\title{
Performance Analysis of Image Steganography based on DWT and Arnold Transform
}

\author{
Shikha Sharda \\ ECE, UIET, Panjab University, Chandigarh
}

\author{
Sumit Budhiraja \\ ECE, UIET, Panjab University, Chandigarh
}

\begin{abstract}
Steganography is the technique that communicates secret data in different carriers like image, audio files etc. in such a way that it is not be visible to attackers. In this paper, performance analysis of image steganography based on Discrete Wavelet Transform (DWT) associated to colored and gray scale images is done. Arnold transformation along with a private key is used during encoding to provide high security. DWT is applied on both the cover image and the secret image and then alpha blending operation is done. Stego-image is obtained by using Inverse Discrete Wavelet Transformation (IDWT) Performance analysis is done by using different wavelet families. The simulation results show that PSNR values of the Arnold Transform based method are better than existing methods.
\end{abstract}

\section{Keywords}

Steganography, Arnold Transformation, Private Key, Alpha Blending, DWT

\section{INTRODUCTION}

Steganography is the study of invisible communication which does not attract the attention of eavesdroppers. Steganography is different from cryptography[1]. In cryptography, the data is transformed into another form which is not understandable by others. Hence results in secure communication. Whereas, steganography techniques hide the existence of the message, so that attacker does not find the location of that hidden message.The main objectives of steganography are imperceptibility, capacity and robustness.

In the last few years, wavelets are being used in most of the steganography techniques. It has been observed that wavelets based techniques are most robust as compared to Least Significant Bit (LSB) based techniques. Also, good quality stego-image is obtained using wavelet based techniques. For image quality measurement, Peak Signal to Noise Ratio (PSNR) is used.

This paper presents DWT based image steganography. Three different wavelets are used in embedding process. Arnold transform is used to scramble the secret image and a private key is applied which provide high security and robustness to the system.

This paper is organized as follows. Previous related work in image steganography is discussed in section 2 . Implementation of image steganography based on DWT and Arnold transformation is presented in Section 3. Simulation results and analysis are presented in Section 4. Finally, conclusions are drawn in Section 5.

\section{RELATED WORK}

Iuon-Chang Lin [2] proposed a steganography scheme with distortion tolerance which uses spatial domain for hiding data. This method provides distortion tolerance and gives better quality of processed image.

A lossless steganography scheme has been introduced that embeds secret data into discrete cosine transformation (DCT) coefficients [3]. This method results in a good image quality. Yih-Kai Lin [4]proposed a steganographymethod that is based on DCT coefficients.

Y.-K. Chan [5]proposed a steganography technique which uses Haar discrete wavelet transformation (HDWT]. This method results in a high capacity and a good image quality.

Kumar [6] proposed an algorithm in which secret message is embed in different coefficients of cover image. PSNR has been used to measure the quality of stego image and embedding in diagonal Detail Coefficients (CD) gives better PSNR as compare to other coefficients.

S. Sarreshtedari [7] proposed a high capacity image steganography. It uses BPCS to determine the capacity of DWT block. Data is embedded in Block rather than bit- plans. This methoduses all the noisy bit-plane for embedding.

N.Raftari[8] introduced Integer Wavelet Transform (IWT) based image steganography.For hiding secret data, assignment algorithm is used which finds the best matching between blocks. It shows high robustness against image processing attacks.

Prabakaran G. [9] proposed a steganography approach which hides large data into small cover image. DWT is applied on both secret and cover images. This DWT based approach provides high security and certain robustness.

H.Motamedi [10] proposed a wavelet based image steganography which use noisy components for embedding the secret information. A threshold is calculated and is used to find the noisy components.

G. Prabakaran [11] proposed a dual transform based steganography. For embedding the secret image within the cover image, a fusion method is used.

\section{IMPLEMENTATION OF IMAGE STEGANOGRAPHY BASED ON DWT}

Image steganography based on DWT uses two processes named as encoding and decoding. In encoding, Arnold transformation is used along with a private key on a secret 
image which gives high security to the algorithm. IDWT is used to obtain stego-image.

In decoding, DWT is applied on both stego-image and cover image.Alpha blending operation is then applied and IDWT is used to get scrambled secret image. Finally, original secret image is obtained with the help of private key.

\subsection{Discrete Wavelet Transform}

The discrete wavelet transform (DWT) decomposes the image into four different bands: LL, HL, LH and HH .For wavelet analysis, it uses filter banks.

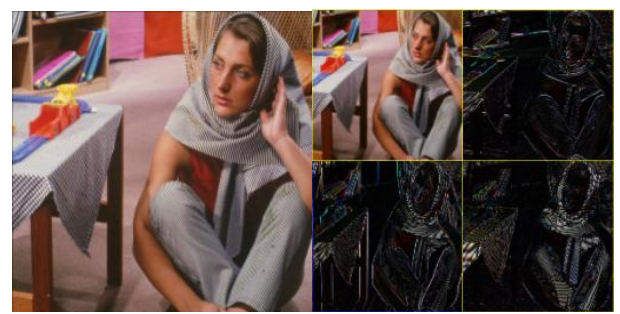

(a)

(b)

Fig 1 (a) Original Lena image and (b) Lena image after Wavelet decomposition

Figure 1 shows single level decomposition of an image using DWT.Different wavelet families are listed in Table.1.

Table 1. List of wavelet families

\begin{tabular}{|l|l|}
\hline Wavelet families & Wavelet name \\
\hline Haar & Haar \\
\hline Daubechies & Db1 \\
\hline Symlets & Sym \\
\hline Coiflets & coif \\
\hline BiorSplines & bior \\
\hline ReverseBior & rbio \\
\hline Meyer & meyr \\
\hline DMeyer & dmey \\
\hline Gaussian & gaus \\
\hline Mexican_hat & mexh \\
\hline Morlet & morl \\
\hline Complex Gaussian & cgau \\
\hline Shannon & shan \\
\hline Frequency B-Spline & fbsp \\
\hline Complex Morlet & cmor \\
\hline
\end{tabular}

\subsection{Scrambling Based on Arnold Transform}

Arnold transformation is proposed by V. I. Arnold in the research of ergodic theory [12].It realigns the pixel matrix of an image. A two dimension Arnold Transform is given as

$$
\left[\begin{array}{l}
x^{\prime} \\
y^{\prime}
\end{array}\right]=\left[\begin{array}{ll}
1 & 2 \\
1 & 1
\end{array}\right]\left[\begin{array}{l}
x \\
y
\end{array}\right](\operatorname{Mod} N)
$$

Where $x$ and $y$ are the coordinates of the original image; $N$ is the order of image matrix; $x^{\prime}$ and $y^{\prime}$ are the coordinate of the transformed image. Thetransform changes the position of two pixels, and if it isdone several times, a disordered image can be generated.

\subsection{Encoding}

In encoding process, DWT is performedon the cover image and secret image is scrambledusing Arnold Transform and then alpha blending operation is applied. IDWT is performed to get the stego-image [9]. Schematically it can be represented as:

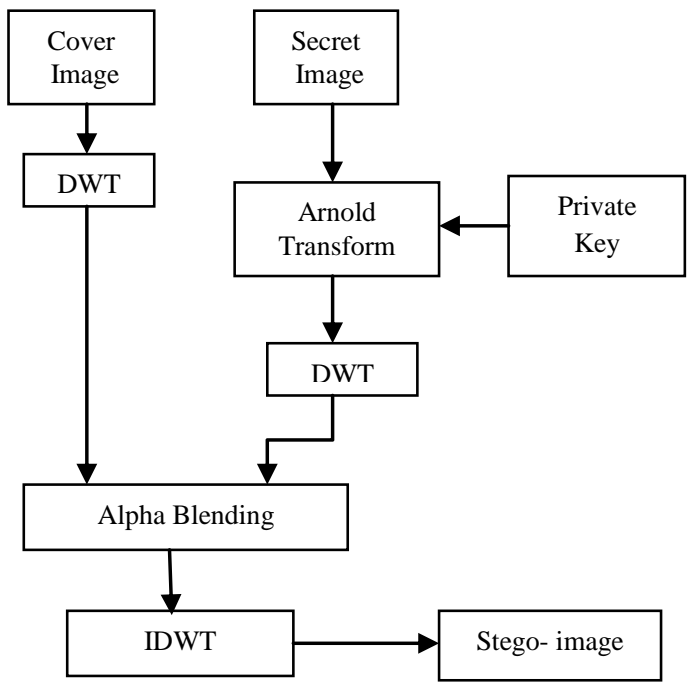

Fig 2: Encoding process

\subsubsection{Encoding Algorithm:}

Step1: (For colored image) The cover image and the secret image are taken and thenthe Red (R) plan is taken separately.

Or

(For grayscale image) The cover image and secret image are taken and then steps 2-7 are performed.

Step2: Single level 2D-DWT decomposition is performed on the cover image.

Step3: A private key with Arnold transformation is applied on image $\mathrm{S}$ and Scrambled Secret Image is obtained (SS).

Step4: Again 2D-DWT at level 1 is performed on the image SS.

Step5: The approximation co-efficient, horizontal coefficient, vertical co-efficient and diagonal coefficient of the stego-imageis obtained as:

Approximation co-efficient of the stego-image $=$ (1-a)*Approximation coefficient of the cover $+a *$ Approximation coefficient of the secret image In the same way, the horizontal co-efficient, vertical co-efficient and diagonal co-efficient of the embedded image are calculated.

Step6: Finally, IDWT is performed to get the R plane of the stego-image (SI).

Step7: For the Green $(G)$ plane and Blue(B) plane,the above method is repeated separately and

Step8: $\quad$ Red $(\mathrm{R})$, Green $(\mathrm{G})$ plane and Blue $(\mathrm{B})$ plane are then concatenated to get the stego-image.

\subsection{Decoding}

DWT transform is performed on both the stego-image and cover image and then alpha blending operation is performed. Next, IDWT is performed to rebuild the scrambled secret 
image. Finally the original secret image is recovered by using the private key [9]. Schematically it can be represented as:

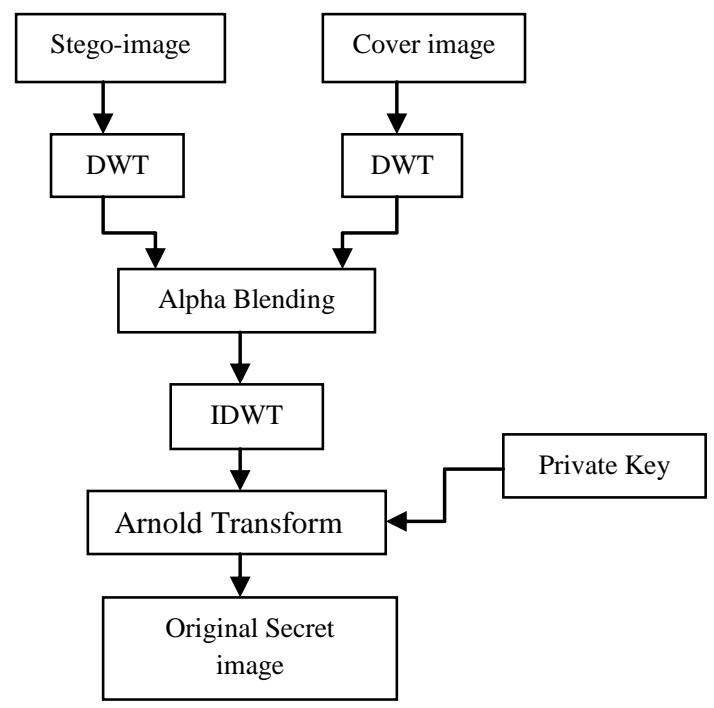

Fig 3: Decoding process

\subsubsection{Decoding Algorithm:}

Step1: (For colored image) The original cover image and the stego-image are taken and then the Red (R) plane is taken separately.

$$
\text { Or }
$$

(For gray scale image) The cover image and stego-image are taken and then steps $2-5$ are performed.

Step2: $\quad$ Single level 2D-DWT decomposition is performed on the cover image as well as on the stego-image.

Step3: The approximation co-efficient, horizontal coefficient, vertical co-efficient and diagonal coefficient of the secret image is obtained as: Approximation co-efficient of the secret image= (Approximation coefficient of the embedded image - (1a)*Approximation coefficient of the embedded image)/a.

In the same way, the horizontal co-efficient, vertical co-efficient and diagonal co-efficient of the embedded image are calculated.

Step4: IDWT is performed to reform the SS

Step5: Finally, Arnold transformation is performed with private key and the $\mathrm{R}$ plane of secret image $\mathrm{S}$ is obtained.

Step6: For the Green (G) plane and Blue (B) plane,the above method is repeated separately and

Step7: $\quad$ Red (R), Green (G) plane and Blue (B) plane are then concatenated to get original the secret image.

\section{SIMULATION RESULTS AND ANALYSIS}

The stego-image after embedding and recovered secret image is shown in Figure 4 and Figure 5. Three different wavelets are used for embedding. Results are obtained by using MATLAB $\mathrm{R} 2012 \mathrm{a}$. The value of alpha is taken as 0.01 .

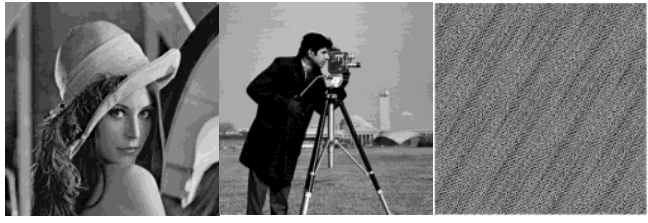

(a)

(b)

(c)

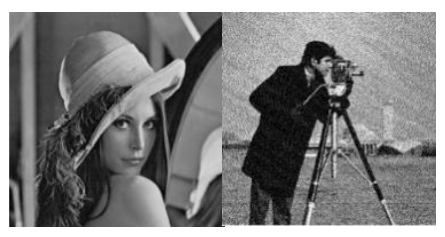

(d)

(e)

Fig 4: (a) Cover image (lena.tif) (b) Secret image (cameraman.tif) (c) Arnold transformation (d) Stego image (e) Recovered secret image

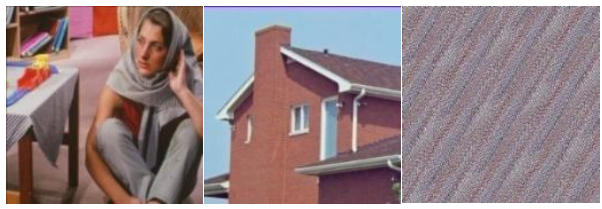

(a)

(b)

(c)

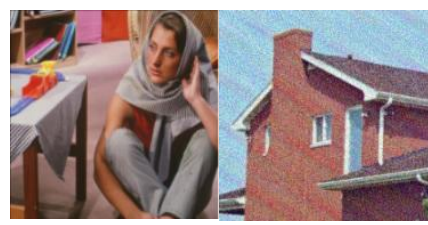

(d)

(e)

Fig 5: (a) Cover image (barbara.tif) (b) Secret image (house.tif) (c) Arnold transformation (d) Stego image (e) Recovered secret image

\subsection{Analysis}

The various image quality parameters are Mean Square Error (MSE), Peak Signal to Noise Ratio (PSNR),Structural Content (SC), Maximum Difference (MD)and Normalized Absolute Error (NAE).These parameters are used to determine the image quality of stego-image.

- $\quad$ Peak Signal to Noise Ratio (PSNR )

- $\quad$ Mean Square Error (MSE)

$$
P S N R=10 \log \left(\frac{255^{2}}{M S E}\right)
$$

$$
M S E=\frac{1}{M N} \sum_{x=1}^{M} \sum_{y=1}^{N}\left(x_{j, k}-x_{j, k}^{\prime}\right)^{2}
$$

- $\quad$ Structural content (SC)

$$
S C=\sum_{j=1}^{M} \sum_{k=1}^{N}\left(x_{j, k}\right)^{2} / \sum_{j=1}^{M} \sum_{k=1}^{N}\left(x_{j, k}^{\prime}\right)^{2}
$$


- $\quad$ Maximum difference (MD)

$$
M D=\operatorname{Max}\left(\left|x_{j, k}-x_{j, k}^{\prime}\right|\right)
$$

- $\quad$ Normalized absolute error (NAE)

$$
N A E=\sum_{j=1}^{M} \sum_{k=1}^{N}\left|x_{j, k}-x_{j, k}^{\prime}\right| / \sum_{j=1}^{M} \sum_{k=1}^{N}\left|x_{j, k}^{\prime}\right|
$$

The original cover image $x$ sized $M \times N$ and the stego image $x^{\prime}$ sized $M \times N$, and $x_{j, k}$ and $x_{j, k}^{\prime}$ are pixel located at the $j^{\text {th }}$ row and the $k^{\text {th }}$ column of images $x$ and $x^{\prime}$, respectively.
PSNR values are measured in $\mathrm{dB}$ and other parameters are calculated in error ratio. It has been observed that for lesser MSE, PSNR is greater. Hence it results into better image quality. Good results are obtained for both colored and gray scale images. The summary of various quality measurements has been given in Table 2 and Table 3.The simulation results are compared with H. Motamedi's [8] andPrabakaran's methods [9]. The PSNR values of stego-images after embedding process for above mentioned methods are given in Table 4 and Table 5.The results reveals that Arnold Transform based method has higher PSNR value than other two methods.

\begin{tabular}{|c|c|c|c|c|c|c|c|}
\hline $\begin{array}{c}\text { Cover image } \\
(256 \times 256) \\
\end{array}$ & $\begin{array}{c}\text { Secret image } \\
(256 \times 256)\end{array}$ & Wavelets & MSE & PSNR & $\mathrm{SC}$ & MD & NAE \\
\hline \multirow[t]{3}{*}{ Lena.tif } & \multirow[t]{3}{*}{ Cameraman.tif } & Db1 & 0.7058 & 49.6441 & 1.0012 & 2.3300 & 0.0071 \\
\hline & & Haar & 0.6805 & 49.8025 & 1.0010 & 2.3200 & 0.0069 \\
\hline & & Bior1.1 & 0.6805 & 49.8025 & 1.0010 & 2.3200 & 0.0069 \\
\hline \multirow[t]{3}{*}{ Monarch.tif } & \multirow[t]{3}{*}{ Cameraman.tif } & Db1 & 0.6380 & 50.0823 & 1.0025 & 2.2200 & 0.0060 \\
\hline & & Haar & 0.6380 & 50.0823 & 1.0025 & 2.2200 & 0.0060 \\
\hline & & Bior1.1 & 0.6380 & 50.0823 & 1.0025 & 2.2200 & 0.0060 \\
\hline \multirow[t]{3}{*}{ Lena.tif } & \multirow[t]{3}{*}{ Monarch.tif } & Db1 & 0.5837 & 50.4692 & 1.0022 & 2.2600 & 0.0063 \\
\hline & & Haar & 0.5837 & 50.4692 & 1.0022 & 2.2600 & 0.0063 \\
\hline & & Bior1.1 & 0.5837 & 50.4692 & 1.0022 & 2.2600 & 0.0063 \\
\hline \multirow[t]{3}{*}{ Lena.tif } & \multirow[t]{3}{*}{ House.tif } & Db1 & 0.6253 & 50.1702 & 0.9980 & 2.2700 & 0.0065 \\
\hline & & Haar & 0.6253 & 50.1702 & 0.9980 & 2.2700 & 0.0065 \\
\hline & & Bior1.1 & 0.6253 & 50.1702 & 0.9980 & 2.1900 & 0.0065 \\
\hline
\end{tabular}

Table2.Various image quality parameters for the stego-images after encoding process (gray scale image)

\begin{tabular}{|c|c|c|c|c|c|c|c|}
\hline $\begin{array}{c}\text { Cover image } \\
(256 \times 256)\end{array}$ & $\begin{array}{c}\text { Secret image } \\
(256 \times 256)\end{array}$ & Wavelets & MSE & PSNR & $\mathrm{SC}$ & MD & NAE \\
\hline \multirow[t]{3}{*}{ Barbara.tif } & \multirow[t]{3}{*}{ House.tif } & Db1 & 0.6336 & 50.3381 & 0.9990 & 2.0833 & 0.0060 \\
\hline & & Haar & 0.6500 & 50.2373 & 0.9991 & 2.0900 & 0.0060 \\
\hline & & Bior1.1 & 0.6508 & 50.2339 & 0.9991 & 2.0667 & 0.0060 \\
\hline \multirow[t]{3}{*}{ Parrot.tif } & \multirow[t]{3}{*}{ Tower.tif } & Db1 & 0.6115 & 50.3090 & 1.0042 & 2.5233 & 0.0056 \\
\hline & & Haar & 0.5998 & 50.3955 & 1.0041 & 2.4233 & 0.0055 \\
\hline & & Bior1.1 & 0.5998 & 50.3955 & 1.0041 & 2.4233 & 0.0055 \\
\hline \multirow[t]{3}{*}{ Barbara.tif } & \multirow[t]{3}{*}{ Tower.tif } & $\mathrm{Db} 1$ & 0.4422 & 51.6788 & 1.0010 & 2.1633 & 0.0050 \\
\hline & & Haar & 0.3913 & 52.2067 & 1.0007 & 2.0433 & 0.0047 \\
\hline & & Bior1.1 & 0.3913 & 52.2067 & 1.0007 & 2.0433 & 0.0047 \\
\hline \multirow[t]{3}{*}{ Tower.tif } & \multirow[t]{3}{*}{ Barbara.tif } & Db1 & 0.4171 & 51.9324 & 1.0039 & 2.1467 & 0.0041 \\
\hline & & Haar & 0.4171 & 51.9324 & 1.0039 & 2.1467 & 0.0041 \\
\hline & & Bior1.1 & 0.4171 & 51.9324 & 1.0039 & 2.1467 & 0.0041 \\
\hline
\end{tabular}

Table 3.Various image quality parametersfor the stego-imagesafter encoding process (colored image)

Table 4. Comparison between H.Motamedi's method and Arnold Transform based method

\begin{tabular}{|l|l|l|l|l|l|}
\hline \multicolumn{1}{|c|}{ Cover image } & \multicolumn{1}{c|}{ Secret image } & \multicolumn{2}{c|}{ H. Motamedi et al. } & \multicolumn{2}{c|}{$\begin{array}{c}\text { Arnold Transform based } \\
\text { method }\end{array}$} \\
\cline { 3 - 6 } & & MSE & PSNR & MSE & PSNR \\
\hline Lena.jpg & Airplane.jpg & 4.37 & 41.37 & 0.7453 & 49.4074 \\
\hline Barbara.jpg & Airplane.jpg & 6.59 & 39.65 & 0.8622 & 48.7749 \\
\hline
\end{tabular}


Table 5. Comparison betweenG. Prabakaran's method and Arnold Transform based method

\begin{tabular}{|l|l|l|l|l|l|}
\hline \multicolumn{1}{|c|}{ Cover image } & \multicolumn{1}{|c|}{ Secret image } & \multicolumn{2}{c|}{ G. Prabakaran et al. } & \multicolumn{2}{c|}{$\begin{array}{c}\text { Arnold Transform based } \\
\text { method }\end{array}$} \\
\cline { 3 - 6 } & & MSE & PSNR & MSE & PSNR \\
\hline Azul.jpg & Wind.jpg & 1.2116 & 47.2971 & 0.6631 & 49.9922 \\
\hline Winter.jpg & Blue hills.jpg & 0.5241 & 50.9368 & 0.4422 & 51.6788 \\
\hline
\end{tabular}

\section{CONCLUSION}

The performance analysis of image steganography based on DWT is done by using different wavelets. This method is associated to both colored and gray scale images. A private key has been used in this algorithm which provides high security to the system. Db1/Haar/Bior1.1 wavelets are used in embedding process.From results, it has been observed that there is not much difference in the values of quality parameters by using different wavelets. But Bior1.1 gives slightly better results in some cases. The stego- image looks unaltered. Hence, results in better quality image. The results show that Arnold Transform based method has higher PSNR values than other existing methods.

In future this method can be tested by using other wavelets and different values of alpha.

\section{REFERENCES}

[1] Nagham Hamid et al. "Image Steganography Techniques: An Overview"International Journal of Computer Science and Security (IJCSS), Volume (6): Issue (3): 2012.

[2] I.-C. Lin et al, "Hiding data in spatial domain images with distortion tolerance", Computer Standards \& Interfaces 31 (2009) 458-464.

[3] C.-C. Chang et al., "Reversible hiding in DCT-based compressed images", Information Sciences 177 (2007) $2768-2786$.

[4] Yih-Kai Lin, "High capacity reversible data hiding scheme based upon discrete cosine Transformation", The Journal of Systems and Software 85 (2012) 2395- 2404.
[5] Y.-K. Chan et al., "A HDWT-based reversible data hiding method", The Journal of Systems and Software 82 (2009) 411-421.

[6] Vijay Kumar and Dinesh Kumar, "Performance Evaluation of DWT Based Image Steganography”, 2010 IEEE 2nd International Advance Computing Conference.

[7] S. Sarreshtedariet al. "High Capacity Image Steganographyin Wavelet Domain"IEEE CCNC 2010 proceedings

[8] N.Raftari, "Digital Image Steganography Based on Integer Wavelet Transform and Assignment Algorithm", 2012 Sixth Asia Modelling Symposium.

[9] Prabakaran. G and Bhavani.R, "A Modified Secure Digital Image Steganography Based on Discrete Wavelet Transform", 2012 International Conference on Computing, Electronics and Electrical Technologies [ICCEET].

[10] H.Motamedi“A New Image Steganography Based on DenoisingMethods in Wavelet Domain" ${ }^{\text {th }}$ International ISC Conference on Information Security and Cryptology 2012.

[11] G. Prabakaran“Dual Transform Based Steganography Using Wavelet Families and Statistical Methods"Proceedingsof the 2013 International Conference onPattern Recognition, Informatics and Mobile Engineering (PRIME) February 21-22.

[12] Lingling $\mathrm{Wu}$ et al."Arnold Transformation Algorithm and Anti-ArnoldTransformation Algorithm"The 1st International Conference on Information Science and Engineering (ICISE2009). 